\title{
Understanding business strategies of networked value constellations using goal- and value modeling
}

\author{
Michaël Petit \\ University of Namur \\ 21 Rue Grandgagnage \\ B-5000 Namur, Belgium \\ mpe@info.fundp.ac.be
}

\author{
Jaap Gordijn \\ VUA Amsterdam \\ De Boelelaan 1081 \\ 1081 HV Amsterdam, The Netherlands \\ gordijn@cs.vu.nl
}

\author{
Roel Wieringa \\ University of Twente \\ P.P. Box 715 \\ 7500 AE Enschede, The Netherlands \\ roelw@cs.utwente.nl
}

\begin{abstract}
In goal-oriented requirements engineering (GORE), one usually proceeds from a goal analysis to a requirements specification, usually of IT systems. In contrast, we consider the use of GORE for the design of IT-enabled value constellations, which are collections of enterprises that jointly satisfy a consumer need using information technology. Designing the constellation amounts to designing the architecture of IT, business processes, and coordination processes across the cooperating companies. The requirements analysis needed to do such a cross-organizational design not only consists of a cross-organizational goal analysis, in which the relevant goals of the participating companies are aligned, but also of a value analysis, in which the commercial sustainability of the constellation is investigated. In this paper we investigate the relation between crossorganizational GORE and value modeling. We use theories about business strategy such as those by Porter to identify strategic goals of a value web, and operationalize these goals using value models. We show how value modeling allows us to find more detailed goals, and to analyze conflicts among goals.
\end{abstract}

\section{Introduction}

As a result of the widespread use of the Internet, enterprises increasingly organize themselves as value constellations: collections of enterprises that jointly satisfy a consumer need, where each enterprise contributes its own spe- cific expertise, products, and services [18].

Since enterprises are profit-and-loss responsible entities, they only will participate in a value constellation if there is a reasonable chance they all make a profit. So, before embarking on a requirements engineering process for the software components at hand supporting such a constellation, it is important that first the value constellation itself is properly understood, and analyzed, e.g. for profitability. Where in 'technical systems' satisfiability is important, commercial sustainability is a first concern for software systems supporting value constellations.

We propose to assess sustainability by distinguishing different views for different stakeholders, each presenting the information that a stakeholder needs for decision-making. In doing so, we distinguish the following stakeholders.

- Business managers who decide whether or not to go ahead with forming the value constellation. For these stakeholders, two views are relevant. First, a model of strategic business goals of each of the participating business actors is important, showing for each actor which strategic, long term, goals it wants to achieve. Second, a model of the desired value exchanges among the business actors is of relevance that realizes the business goals as represented in the goal model. This value model shows which goods and services the actors deliver to one another and to the consumer whose need is to be fulfilled. It shows how the goals represented in the goal model are to be realized by exchanges of commercial value.

- Business architects who design business processes and 
responsibility structures. For these stakeholders, a process model is relevant that shows how businesses cooperate to realize the value exchanges as represented in the value model. Where the value models only show which objects (services or products) of value are exchanged, the process model shows how this is done. It shows coordination needed across the business actors, and the business processes performed by each actor to realize this coordination.

- IT architects who design IT support for business processes. For these stakeholders, information system architecture models are relevant.

Business consultants, who help businesses to form a value constellation, must discuss design options with different stakeholders. The discussion with each stakeholder will take place within one of the above viewpoints. In this paper we focus on the viewpoint of business managers. We will investigate how strategic goals can be modeled, and then can be mapped on a value model of a business cooperation. We will find that there is a mutual interaction between these two activities: Value modeling usually identifies new business actors and thus new strategic goals of these actors not identified in goal modeling in the first place. This forces the consultant to move back and forth between goal modeling and value modeling to mutually align these two models.

\section{Modeling value constellations: Require- ments}

Restricting ourselves to be business manager's viewpoint, we need to find strategic business goal models and value models. The purpose of this modeling effort is twofold: (1) to create a shared understanding of the various business strategies and value constellations at hand, and (2) to analyze a business strategy and its operation in terms of networked value constellation for economic sustainability.

Gordijn and Akkermans [8] have specified requirements for modeling approaches in the business manager's viewpoint. First, a modeling approach should be lightweight. Strategic goal and value constellation modeling is often an iterative activity. Moreover, since value constellation modeling is often done for a new service or product at hand, it should be done within a reasonable time frame (typically a few weeks), due to time-to-market considerations.

Second, as argued in [15], a business modeling approach should have a graphical syntax.

Third, a modeling approach should enable analysis of economic sustainability. While designing a businesses strategy and constellation for provisioning e-services, the first and foremost goal is that each participating enterprise can create a profit in a sustainable way. For consumers, they should increase their economic utility by obtaining an e-service.

\section{Modeling strategic goals for value constel- lations}

\subsection{A simple goal language for modeling strategic business-related objectives}

For goal models, we extend the above requirements with the following two.

- First, from a design perspective, a goal model should allow business managers and consultants to analyze the coherence of the goals (e.g. to avoid goal conflicts [20]) of the businesses participating in a value constellation and to check, informally, that they can be satisfied by implementing a value model.

- Second, from an evaluation perspective, the goal model should help in understanding why goals are met or not met, by describing causal relations among goals, and to determine whether a value model implements the strategic goals of the various actors involved.

To satisfy these requirements, we have defined a set of goal modeling concepts based mainly on $i^{*}$ and Tropos [1]. It is not our intention here to define a new language, but to define a small set of concepts usable in strategic business goal modeling.

Actors: Actors are enterprises and end consumers that have goals. In the case of enterprises, one of the main goals is economic sustainability. Goals of end consumers are related to the economic utility of the consumption of a product or service.

Goal: As a first approximation, we consider a goal as a condition on a state to be achieved or maintained ${ }^{1}$ or an action to be performed. We believe that this simple concept allows describing most if not all of strategic goals. The notion of state will be explained below into more details.

And/Or, Some+/- Links: Various kinds of means-ends links between goals.

The definition of a goal refers to a notion of state that requires to be defined more precisely. This requires the following additional concepts:

State: We consider a state to be the association of a value to a property (a measure or valuation of that property).

\footnotetext{
${ }^{1}$ Many refinements of this definition are possible. See e.g. the taxonomy of goals proposed in KAOS [4], including classes such as achieve, maintain, avoid, cease and optimize.
} 
Property: A property is some variable that is of interest in the business context and whose state can change over time.

Value: A value is an identifiable element within a scale.

Scale: A set of values that may be associated to a property. It can be of different kinds, such as a nominal scale (without order) or an ordinal scale (ordered). Different scales can be associated to different properties.

Causal relationship: A causal relationship describes that the values of one or more properties influence the value of another property. Many kinds of influences may be defined.

We believe that it is important to make a clear distinction between the description of properties and the causal relationships existing between them and the description of goals and their relationships, for two reasons:

- The causal relationships between properties are stable and exist independently of the goals of actors at a given moment. For instance, profit is always computed from cost and income and their respective influences in this computation are always negative and positive. While the goals of a company with respect to profit, cost and income may change with time, the causal relationship between these properties are stable and do not depend on time. As such, they represent domain knowledge in an indicative mode, whereas goals represent information in an optative mode [11].

- The concepts required for expressing relations among goals and relations among properties are not the same. For example, while a positive causal relationship between cost and profit means that a decrease in the value of cost will increase the value of profit, the fact that the objective of decreasing cost is achieved does not necessarily means that the achievement of the objective of attaining a sufficient profit is increasing (it might even never be effectively achieved).

Though a specific notation for representing causal relationships among properties would be a useful tool in a goal modeling approach, in this paper, we will limit ourselves to represent goal models, using the set of concepts described at the beginning of this section. For these goal models, we will use a subset of the $i^{*}$ notation. It is important to note that in doing this, our intention is not to define a dialect of $i^{*}$. It is just to use a graphical syntax that is a subset of the one of $i^{*}$ for representing the concepts of our proposed simple strategic goal language. The meaning of the graphical elements of $i^{*}$ that we use is not intended to be the $i^{*}$ semantics. This is purely a syntactical use of the $i^{*}$ graphical notation and the semantics of the graphical symbols should be understood as defined at the beginning of this section. The subset of the notation that we use is the following. Actors are represented as a name in a circle and have a boundary represented with a dotted circle. A goal is represented as a rounded box. The links are labeled with the appropriate type (And, Or, Some+ and Some-).

\subsection{Related work on goal modeling}

Current work on goal-oriented requirements engineering (GORE) focuses on formalization and expressiveness of goal modeling notations [12]. KAOS [13] uses temporal logic to analyze goal and resolve conflicts. Its formal nature makes it very appropriate for the requirements engineering of applications where safety requirements are strong and hence verification is required. However, in our case we are interested in strategic business goals and their relationships. $i^{*}$ uses a rich notation to express relations among goals, activities and actors $[3,14,21]$ that can lead to very complex models. Tropos $[7,5,6]$ formalizes this to allow satisfiability analysis.

As a corollary to their expressiveness or appropriateness for a specific purpose, the above cited notations provide a large set of concepts with precise and refined semantics, with the consequence that they demand more effort to be learned. However, for our purpose, we need simple goal models that can be understood by business managers without a long preliminary learning.

\section{Value modeling}

In this paper, we model and analyze a value constellation, consisting of enterprises, and implementing business strategies of these enterprises, using the $e^{3}$-value technique. To make this paper self-contained, we briefly introduce the $e^{3}$-value modeling concepts below, see for a more detailed explanation [8].

Actor: An actor is perceived by his/her environment as an economically independent entity.

Value Object: Actors exchange value objects. A value object is a service, a good, money, or even an experience, which is of economic value for at least one of the actors.

Value Port: An actor uses a value port to provide or request value objects to or from other actors.

Value Interface: Actors have one or more value interfaces, grouping value ports, and showing economic reciprocity. Actors are only willing to offer objects to someone else, if they receive adequate compensation in return. Either all ports in a value interface each precisely exchange one value object, or none at all. 
Value Exchange: A value exchange is used to connect two value ports with each other. It represents one or more potential trades of value objects.

Market Segment A market segment breaks actors into segments of actors that assign economic value to objects equally. This construct is often used to model that there is a large group of end-consumers who value objects equally.

Value Activity: A actor performs one or more value activities. These are assumed to yield a profit.

Dependency path: A dependency path is used to reason about the number of value exchanges in an $e^{3}$-value model. A path consists of consumer needs, connections, dependency elements and dependency boundaries. A consumer need is satisfied by exchanging value objects (via one or more interfaces). A connection relates a consumer need to an interface, or relates various interfaces of a same actor. A path can take complex forms, using AND/OR dependency elements taken from UCM scenarios [2]. A dependency boundary represents that we do not consider any more value exchanges on the path.

Given an $e^{3}$-value model, attributed with numbers (e.g. the number of consumer needs per timeframe and the valuation of objects exchanged), Net Value Sheets (NVF) can be generated (for a software tool see http://www.cs.vu.nl/ $\sim$ gordijn/tools.htm). Such sheets show the net cash flow for each actor involved and are a first indication whether the model at hand can be commercially successful for each actor.

Additionally, a series of $e^{3}$-value models can be constructed, modeling how a value model evolves over time. Each value model represents then a snapshot at specific point in time (say on an yearly basis). For such a series, accepted calculations such as Discounted Net Present Value (DNPV) [10] can be done to assess economic sustainability on a per actor basis.

Recently, we have related $i^{*}$ goal-modeling with $e^{3}$ value modeling. In that approach $i^{*}$ models are used to represent a variety of goals, including operational goals [19]. In the current paper, we focus on relating value modeling with strategic business goals, which is why we here use a lightweight subset of $i^{*}$.

\section{Using goal modeling and value modeling: A case study}

\subsection{The Amsterdam Times}

To explain the interplay between strategic goal modeling and value constellation modeling we use a well docu- mented case study we reported on in [8]. This case study is about a consortium of enterprises that want to offer an online news provisioning service to customers. In the reported case study we focus on just the value constellation; in this paper we extend the analysis to strategic business goals.

The case study is about a publisher of news titles. These titles can be seen as independent news papers (just paperbased, daily news papers). The publisher sees these titles as profit-and-loss responsible business units, and so these titles can decide on their own about business issues. The purpose of the publisher is to share facilities that require economies of scale, such as printing, logistics and IT, and to share facilities related to personnel, finance, etc.

For the publisher, one of us (Gordijn) acted as consultant to create new business. In the following sections, we split our activities into problem analysis and solution analysis.

\subsection{Problem Analysis}

\subsubsection{Strategic goals: Economic sustainability and in- crease of economic utility}

We start our problem analysis with two top-level strategic goals, which are broadly accepted from economics. First we suppose that businesses have the strategic goal to be economically sustainable. Second, we assume that final consumers have the goal to optimize economic utility given their budget constraints. These goals are sufficient to create an initial shared understanding among the participating businesses, and we now turn to value modeling to see if we can find a value constellation that realizes these two goals.

\subsubsection{Value model}

To understand the value constellation the titles and a publisher are part of, we create an $e^{3}$-value model (see figure 1). There are many readers that buy a newspaper (e.g. using a subscription) from a title they select. In this constellation, all the titles obtain services (e.g. printing services) from a publisher and pay a fee in return. The titles obtain also fees from advertisers, who pay for publication of their ads in the physical newspaper of a title. As the dependencies show, the amount of money to be paid by the advertiser to the title, relates to number of readers - as for each reader, money has to be paid for an ad.

If we attribute the $e^{3}$-value model with numbers, we can assess economic sustainability for each actor involved (see section 4). Suppose now that we find that according to the best of our estimations, the profitability of the titles and the publisher decreases (in the long run). We now return to goal modeling to understand why this can happen. In the next section, we elaborate on the two goals identified earlier. 


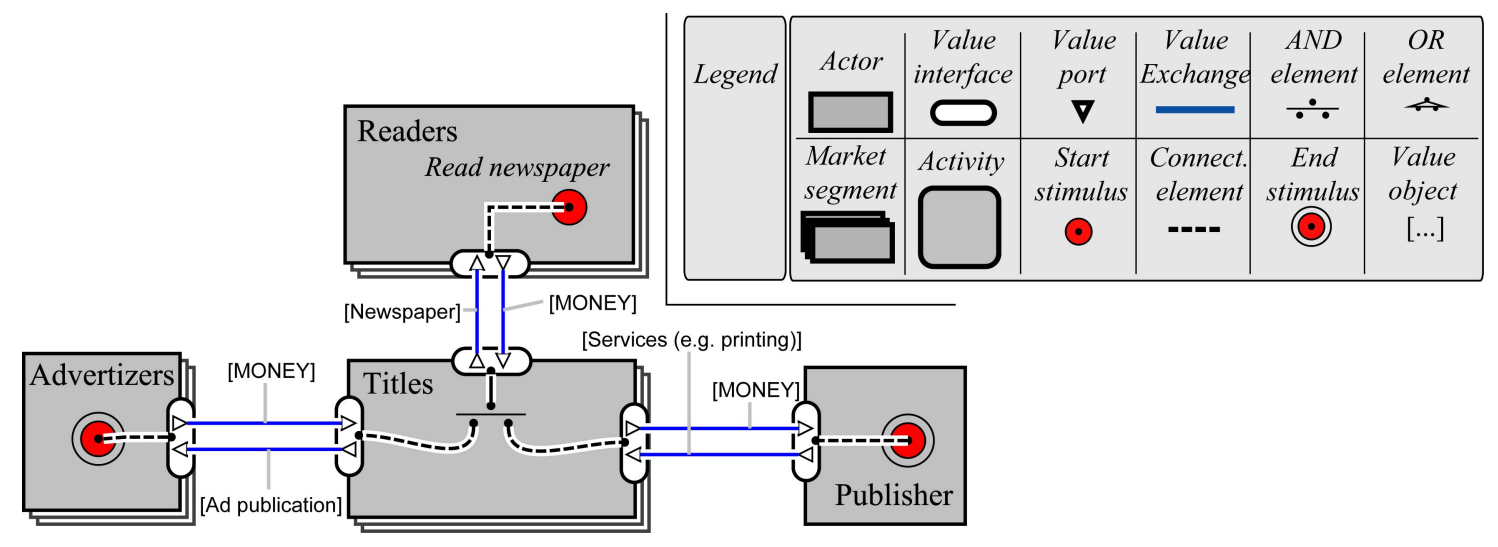

Figure 1. Value model for problem analysis

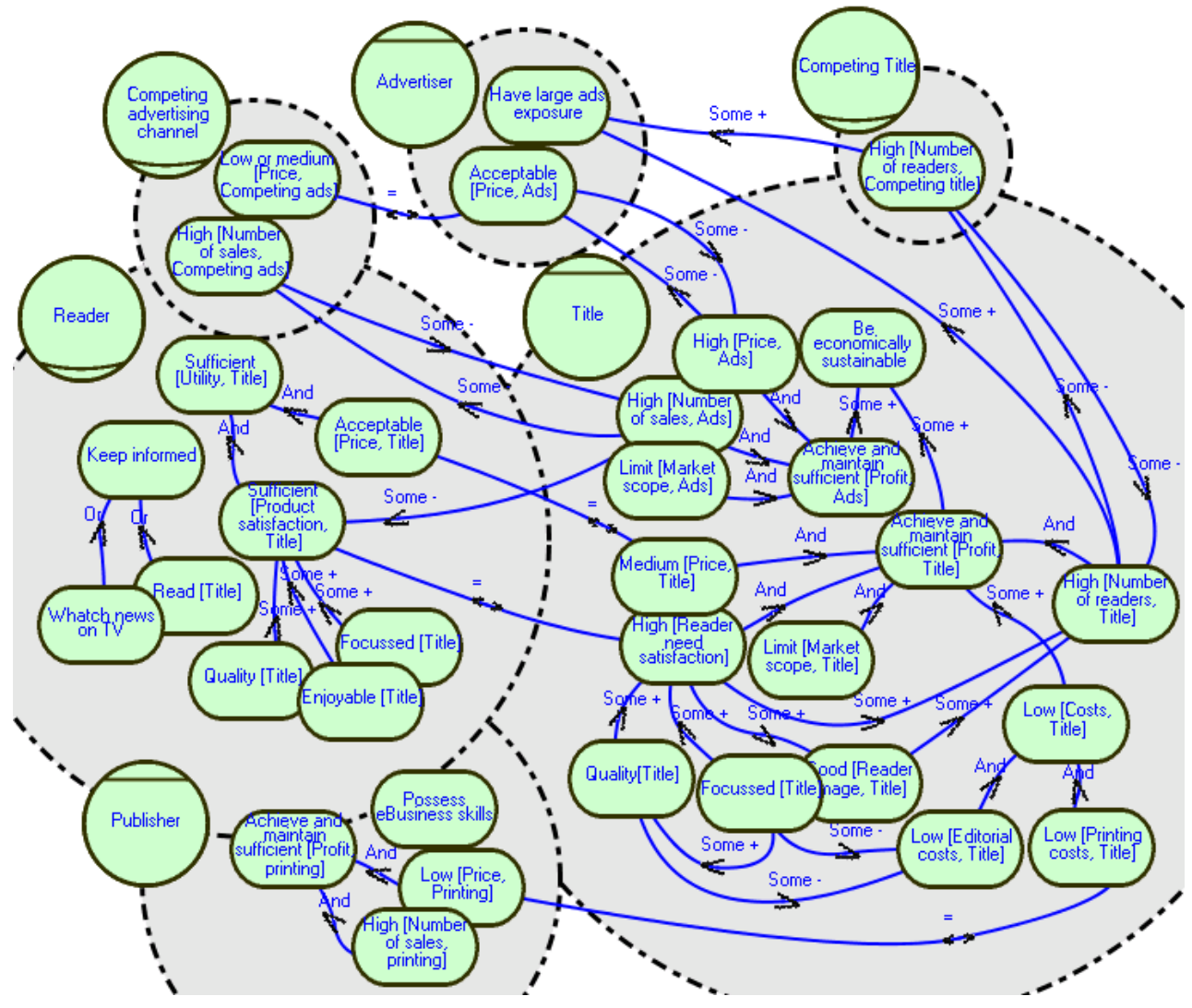

Figure 2. Goal model for problem analysis

\subsubsection{Goal model}

Figure 2 shows why profitability decreases as explained in section 5.2.2. It depicts the involved actors and shows for each of them his "personal" strategic goals. The central actor is the Title. Two actors can be considered as consumers of the Title, namely the Reader who consumes the newspa- per, and pays for doing so, and the Advertiser who pays for placing ads. The last actor, the Publisher, has goals similar to any other company (mainly economic sustainability) but due to lack of space, its objectives are not detailed in our model. 
Reader and advertiser objective. We have elicited the goals for the reader using the consumer value theory of Holbrook [9]. Holbrook defines eight classes of consumer needs, which we use to identify possible consumer goals. The classes are defined based on three differentiating criteria, namely (1) the intrinsic or extrinsic nature of the value of the consumed product or service, (2) the active or reactive nature of the consumption of the product or service and (3) the self- or other-oriented nature of the consumption. This leads Holbrook to identify the following eight classes of consumer needs: efficiency, play, excellence, aesthetics, status, ethics, esteem and spirituality.

Applying this to identify consumer goals our case, we find that the Reader's goals in the consumption of the title newspaper are mainly of the efficiency category (using the newspaper not for itself but rather to achieve another objective, which is to Keep informed ${ }^{2}$ ) and of the play category (personally and actively enjoying the characteristics of the product, described as the two goals Enjoyable [Title] $]^{3}$ and Focused [Title $]^{4}$ ).

In all cases, costumers will balance the value brought to them by the product (described by the above mentioned categories) and the monetary flow that they have to exchange to benefit from the product or service. This always leads to some goal that the price remains acceptable (Acceptable [Price, Title] in the case of the reader).

Title goal model and strategies. The Title as any company has as main goal to Be economically sustainable and has for this two sub-goals, related to the two products/services it puts on the market: Achieve and maintain sufficient [Profit, Title] and Achieve and maintain sufficient [Profit, Ads]. Both of these have a positive effect on economic sustainability but none of then is neither necessary nor sufficient to achieve the latter objective, hence, the use of Some+ links rather than And links.

With respect to elicitation of sub-goals and objectives, we use the well-known theory of Porter [17]. Porter distinguishes two possible strategic goals. First, in case of low cost leadership, an enterprise tries to produce its good or services with the lowest possible costs in the industry. This often results in a standard product or service, of which many units can be produced against low unit costs, by reaching economies of scale. Second, in case of differentiation, an enterprise tries to distinguish itself from its competitors, e.g. by adding extra services to the core product. The claim of Porter is - as he shows in many case studies - that successful enterprises should choose for only one of these goals.

\footnotetext{
${ }^{2}$ Some readers may also have some more specific objectives (e.g. an investor needing a financial title for preparing investment decisions).

${ }^{3}$ If the newspaper contains, for example, some relaxing news or crosswords.

${ }^{4}$ The correspondence of the news topics with the interests of the reader is a source of satisfaction for the reader.
}

So, if companies show signs of trying to reach both types of goals, there is a goal conflict. (Actually, Porter distinguishes a third goal focus, e.g. on a specific region. This goal can be used in conjunction with low cost leadership and differentiation.)

Regarding the first sub-objective (profitability of title), the company adopts a strategy mainly based on differentiation. Due to the already low cost strategy of competing newspapers and the difficulty to target at a homogeneous market (readers are often interested in local news, or news colored by some life-philosophy such as a specific religion), a strategy based on cost leadership is not applicable. Titles can acquire and keep readers only by maintaining a strong focus on the readers' needs and preferences and on the quality of news. This requires amongst others that the title produces focused news (local and/or politically or phylosophically colored). This strategy is represented by decomposing the title sustainability objective in 4 sub goals, with an And decomposition: High [Reader need Satisfaction, Title], Limit [Market scope, Title], High [Number of reader, Title] and Medium [Price, Title].

Due to space restrictions, we can not elaborate on the objectives of the Advertiser, but a similar reasoning can be applied to the Advertiser to elicit its objectives.

Internal actor goal conflicts: Conflicts in strategy and value propositions . While constructing the goal model, we found that some goals relate to the overall goal of low costs. This results in internal actor goal conflicts, due to Porter's theory (choosing low cost leadership and differentiation is called by Porter 'stuck into the middle'). As an example, the goal model shows that the title tries both to achieve news quality while focussing at the same time at reducing costs such as e.g. the editorial costs. However, these objectives might be conflicting since focused and quality news requires a larger number of specialized and skilled journalists, which has a higher cost than e.g. relying on more generalist journalists to produce general news that would have a large diffusion. However cost is still a concern for the title ${ }^{5}$ and has lead to outsourcing of a number of services to the publisher (only the cost for printing is represented).

The goal model also allows the representation of conflicts between various value propositions. In our case, a conflict exists between the goal of the title to display a large quantity of advertisement in its title editions (High [Number of sales, Ads]) to ensure the profitability of the ads service, and the goal to achieve sufficient costumer satisfaction (High [Reader need satisfaction]) since the more ads are present in the newspaper, the less value for the reader.

\footnotetext{
${ }^{5}$ See the Low [Cost, Title] goal linked to the title profitability objective with a Some+ link rather than a And link, since it is not an essential part of the strategy.
} 
Goal conflicts between actors: Competition. As described in section 5.2.2, the economic sustainability of the title is not ensured. The goal model of figure 2 can help to describe why, by adding additional actors describing the competitors of the titles and the strategy adopted by them. This is an addition to $e^{3}$-value analysis, because $e^{3}$-value does not consider competition at all. Nevertheless, in analyzing competitive advantage, competition is an important factor to consider [16]. The title faces competition by two kinds of actors: Competing titles and Competing advertising channels. These actors can be analyzed and modeled according to their objectives and strategy and the consequences on the objectives of the title can be described. For instance, the situation with competing titles shows that they have similar objectives as the title (High [Number of readers, competing title]) and that this goal conflicts with the same objective of the title (represented as dual Some- links among the two goals).

Discussion. The interplay of goal- and value modeling learns a few lessons. First, the value model is used to show that economic sustainability is not reached, whereas the goal model shows why this is the case, e.g. by conflicting goals. Second, although we have not defined a formal relationship between goal- and value models, there can be some relationships observed. The actors in the goal model correspond to actors in the value model, and the top-level goal - economic sustainability - corresponds to the result of value model analysis on DNPV. Finally, the diagnosis based on the goal models directs management to find possible solutions. In the next section, we explore solutions for lack of sustainability, again by constructing goal- and value models.

\subsection{Solution analysis}

A possible (partly) solution to insufficient economic sustainability is to find new services that contribute to profit. Finding new, innovative, services is a creative and difficult task. One of the participants of the publisher's title came up with the suggestion that there is a vast amount of unpublished news articles. Journalists produce more articles than can be published in a newspaper (due to physical restrictions). In addition, there exists an (electronic) archive of all articles ever written by the journalists of the titles. The idea proposed is to make this archive online available as an additional service to newspaper subscribers for free, thereby differentiating from competitors. We first present a goal model for this solution (section 5.3.1), and then show a possible $e^{3}$ value model that implements this solution (section 5.3.2). As the value model shows, new actors are required to offer an online news article service, so we construct a new goal model (including the new actors) in section 5.3.3, to ana- lyze whether goal conflicts between the (new) actors exist that can inhibit a successful execution of the new service.

\subsubsection{A goal model for an online news service}

The title wants to differentiate from its competitors and will provide an online news edition, for instance for readers who need news for their work (need to copy/paste or reference texts in memos) and who are frequently traveling and need to read news from abroad). All this is reflected by the change of the previous goal High [Reader need satisfaction, Title] of the title into the goal Increased [Reader need satisfaction, Title] (see Figure 3).

On-line news has advantages for these readers, which fall mainly in Holbrook's efficiency category. This is explicitly reflected in the goal model by, for instance, adding goals to the reader such as Exploit news texts that shows an additional utility of the news for the reader, but also the Low [Effort, Exploit news text, Any title] since on-line news can easily be referenced in memos or copied into other documents and High [Accessibility, Any title] since the on-line contents can potentially be accessed from anywhere on the internet (for those readers who travel a lot).

Regarding advertisers, the on-line news product could also be used by the title to improve its advertising service (Increased [Advertised needs satisfaction]). The costumers of the service will be readers with a more specific profile than the usual profile than the previous readers: those interested in using Internet based products and who have a need to exploit news for their work. Consequently, this might help the advertisers to better target their ads at these costumers (see the Target at most probable costumers) goal added to the advertiser.

Finally, the title has yet a concern of keeping its operating costs low. As the publishing of on-line news represent an additional cost, this cost should be kept as low as possible not to endanger the profitability objective. This Low [Online Publishing cost, Title] goal will be a determinant in defining a new value model, involving additional partners as described in the following section.

\subsubsection{A value model for an online news service}

While designing an $e^{3}$-value model that implements the goals Increased [Reader need satisfaction, Title], Increased [Advertised needs satisfaction] and Low [Online Publishing cost, Title], various choices still can be made [8]. Here we discuss one possibility (see figure 4). During the process of finding a solution, it rather early showed up that the titles and the publisher lack sufficient capabilities for executing all technical operations (e.g. hosting a website and an article database for a large number of subscribers, and ensuring that each subscriber has access to this site and database). 


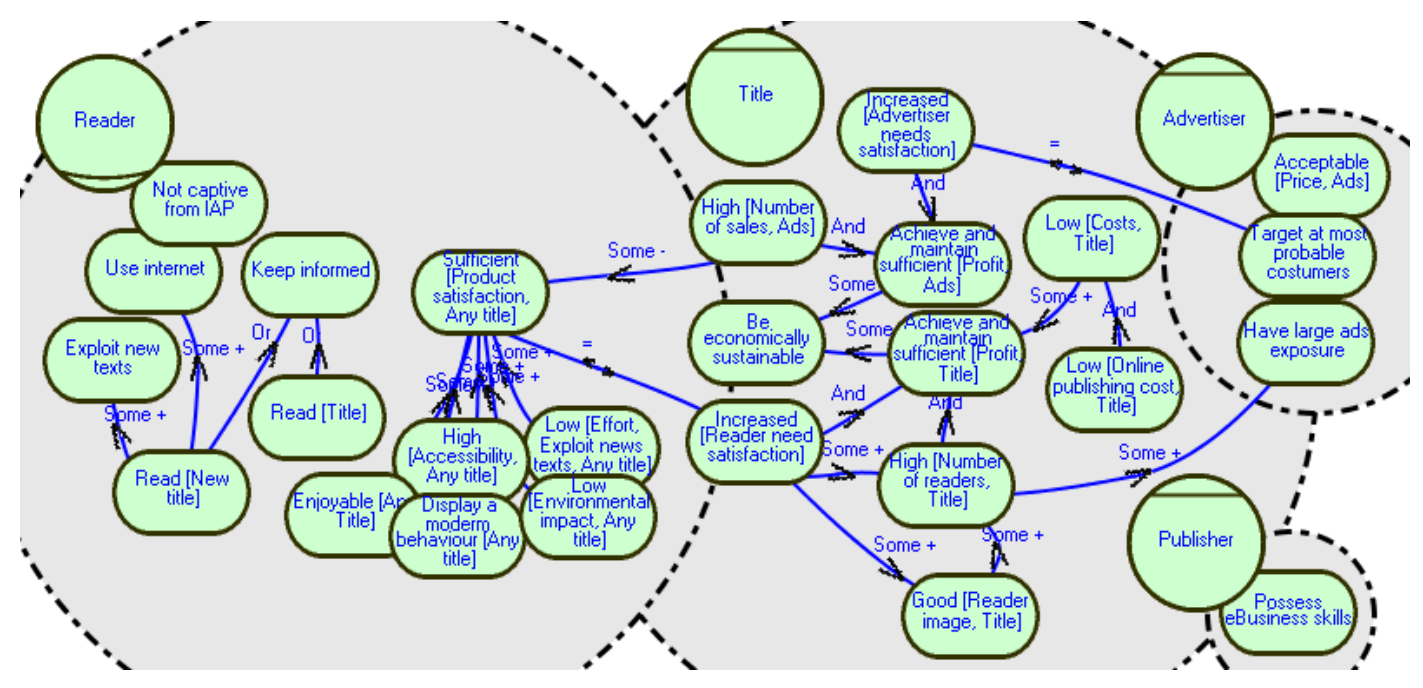

Figure 3. Goal model for the new value proposition

As it was requested that online service comes into operation quickly, it was decided to extend the consortium (at that time consisting of titles, a publisher and advertisers) with an Access Provider (AP) (to offer Internet access for subscribers) and with a Hosting Provider (HP) (to host a large scale article database and website).

An additional requirement was that the online article service is really an additional free service to the subscription of newsreaders on a title. The goal was to differentiate from competitors, by adding an additional service. However, as we have seen in the problem analysis, economic sustainability is a problem a long term. The article online service should help in attracting and keeping subscribers on titles, but this should be done against as low costs as possible.

Figure 4 shows how this low-cost requirement is addressed. The title and the AP jointly offer an online article service to subscribers. The AP delivers broadband Internet access and the subscriber pays for this. Additionally, the title provides the online article and in return obtains 'traffic', which means utilization of the AP's network. At the back, this utilization of network capacity is re-sold, via the publisher, to the AP. What happens is that titles cause substantial network traffic by using their content and their subscriber base. The publisher aggregates this traffic from all titles and arranges a deal with the AP, such that each title gets paid for generating this traffic. This closely corresponds to the goal of the publisher: low cost leadership. As a result, the titles can finance this extra service by the revenues paid by the AP. Such a model is called 'terminating revenue-sharing' in the world of access providers. A downside of this model is that is only available for subscribers that obtain their broadband access from one of AP's the publisher has a deal with. As can be seen from the value interface of the title-AP partnership, an online article can only be obtained, if the reader uses the AP in the partnership.

For this model, again DNPV calculations can be made for assessing economic sustainability, of the online article service itself, as well as its contribution to the overall sustainability of the titles and publisher. Additionally, alternative models are possible, such an 'originating revenue sharing' model [8]. Due to lack of space, we can not deal with these topics here.

Discussion. From this modeling cycle, we can learn that to make the new value model, we need knowledge about technical realizations (e.g. whether we out source ITservices or not). This means that the goal and value models generate the first requirements for the IT realization. Without these requirements, the IT part of the solution runs into the danger of not satisfying the business strategies nor contributing to sustainability. Also, the new value model forces us to back to the goal model and include new actors, with their own sustainability goals. So goal- and value modeling are closely iterative. Commercial sustainability computations provide a new angle on goal modeling, not present in satisfiability checking. The question in our approach is not whether a set of devices and people can satisfy the goals (as in the KAOS modeling of the San Fransisco BART) but whether a set of businesses can satisfy the goals, using their IT systems. This is the novelty of our use of goal modeling.

\subsubsection{Goal models revisited}

Because new actors come into the value constellation (as discovered by the previous step), we have to reason about 


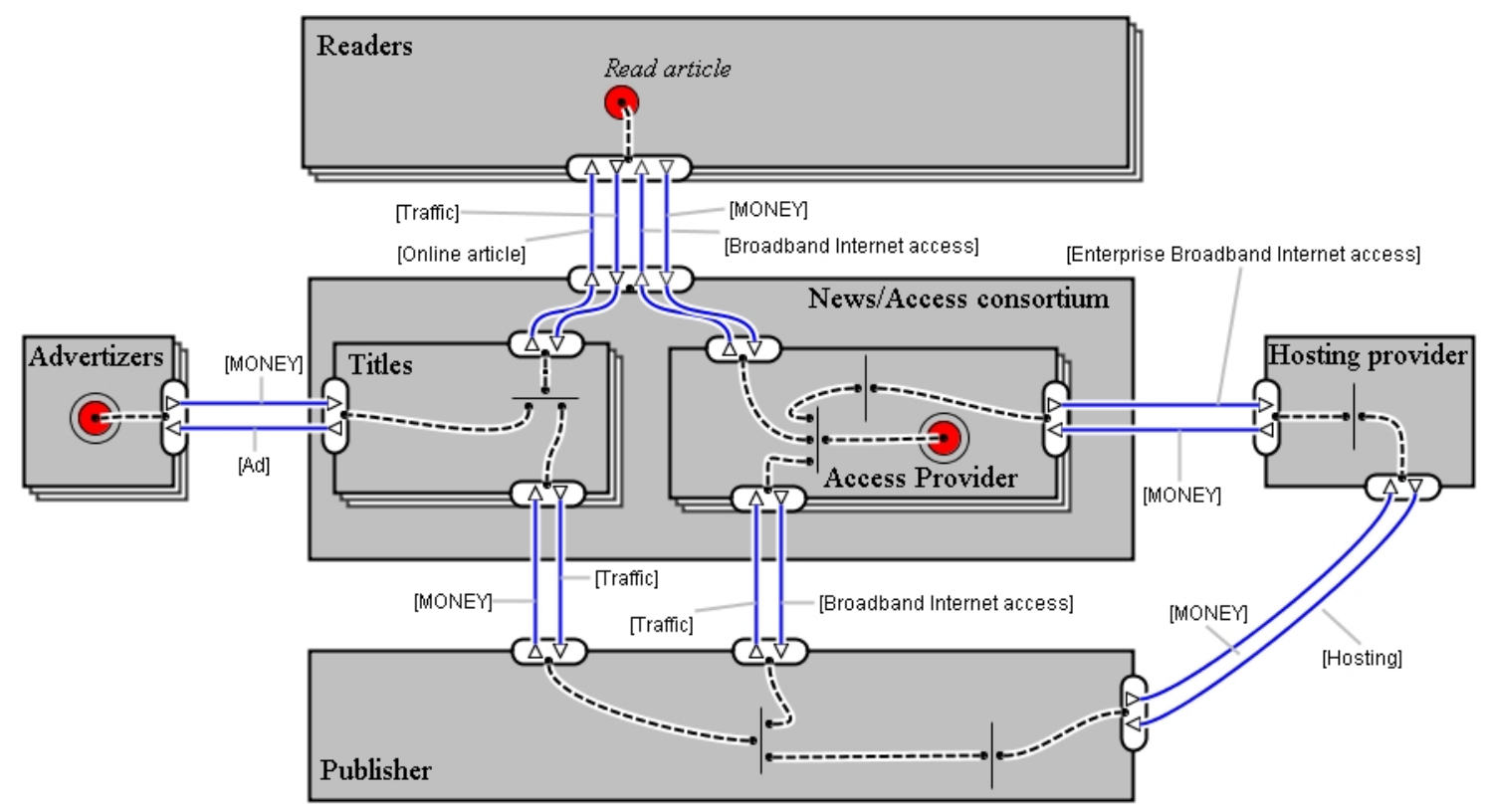

Figure 4. A value model for provisioning online articles using revenue sharing

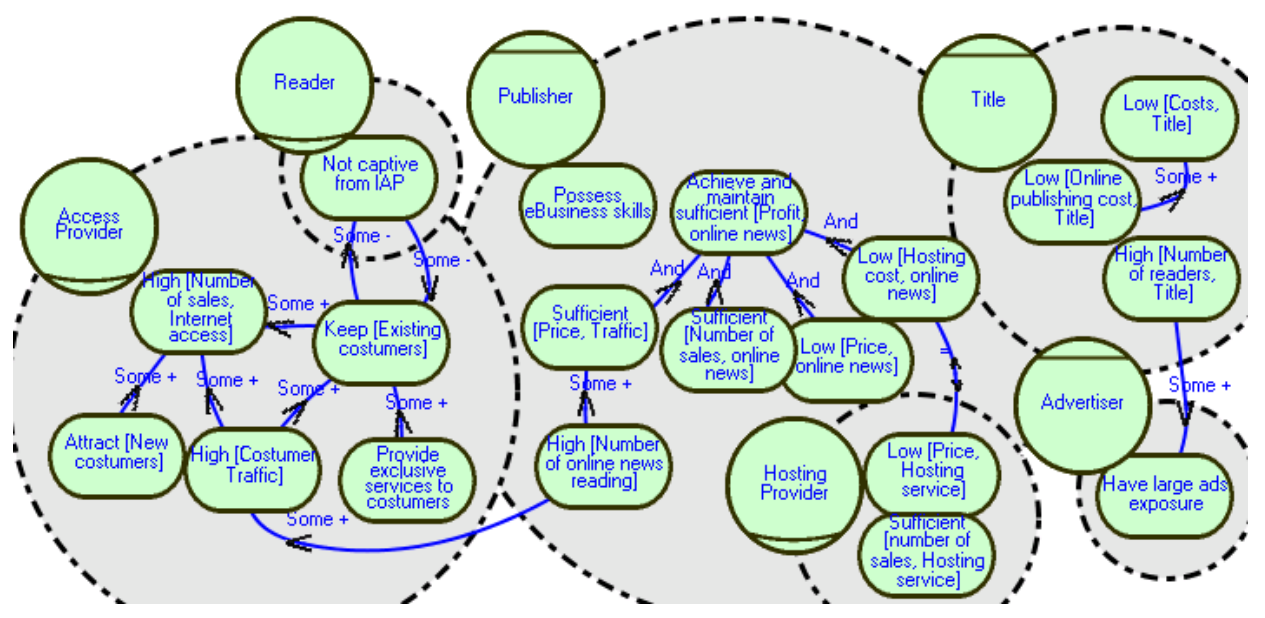

Figure 5. Goal model of the new value constellation

their goals. The modified goal model in figure 5 shows the objectives of the Access Provider. One of the main concerns of this actor is to keep a large market share in the current environment characterized by strong competition (High [Number of sales, Internet access]). To meet this objective, the access providers tries both to Keep [Existing costumers] and to Attract [New costumers] by differentiating from other providers. One way of doing this is to Provide exclusive services to costumers. This is one of the motivations for participating in the value constellation because the free on-line articles of the titles will be available only to those costumers who buy a subscription with an Internet Access of the concerned access provider.

The goals of the publisher are conformant with its current cost leadership strategy. It seeks to provide the on-line news publishing service to the titles at a low price and at low cost (see the Achieve and maintain sufficient [Profit, Online news] goal and its And decomposition into four sub objectives. One of these goals is to get sufficient revenue from reselling the Internet traffic to the access providers (Sufficient [Price, Traffic]). In order to ensure this, the publisher needs to ensure a High [number of online news reading], 
in order to get a reasonable bargaining power against the access providers. For this, he will depend on his service consumption by the titles and ultimately by the title readers themselves.

As the goal model shows through a Some+ link, this last goal is in-line with a goal of the access provider (High / Costumer Traffic]). The access provider considers that the high consumption is good because it helps both in keeping the existing costumers and in attracting new ones (since costumers might encourage non-costumers to buy the service).

From this second goal modeling step, we can see that, the goal model can help in taking into account, by making them explicit, the motivations and objectives of the value constellation participants. This might in some case lead to refinements of the value model and possibly to rule out some of the envisaged models.

\section{Conclusion}

We have presented some insights on the benefits of using an approach based on the combined use of goal and value modeling for early requirements engineering of business and e-business systems. We use theories about business strategy such as those of Porter and Holbrook to identify strategic goals of a value web, and operationalize these goals using value models. We show how value modeling allows us to find more detailed goals, and to analyze conflicts among goals. In particular, we show how value modeling can be used to support decisions about trade-offs among conflicting goals.

The approach is original because it concentrated on goals at a very strategical level rather than at an IT "system" level. The approach presented in this paper is a step in proposing solutions for better alignment of IT systems with the strategy of companies.

Possible future research directions include a better formalization of the link between the goal model and the value model, the link between the value model and more detailed business process models, and ultimately the link to software systems.

\section{References}

[1] P. Bresciani, A. Perini, P. Giorgini, F. Giunchiglia, and J. Mylopoulos. Modelling early requirements in tropos: a transformation based approach. In P. Ciancarini, M. Wooldridge, and G. Weiß, editors, Agent-Oriented Software Engineering II, pages 151-168. Springer. LNCS 2222.

[2] R. J. A. Buhr and R. S. Casselman. Use Case Maps for Object-Oriented Systems. Prentice Hall, Englewood Cliffs, NJ, 1999.

[3] L. Chung, B. A. Nixon, E. Yu, and J. Mylopoulos. NonFunctional Requirements in Software Engineering. Kluwer, 1999.
[4] A. Dardenne, A. v. Lamsweerde, and S. Fickas. Goaldirected requirements acquisition. Science of Computer Programming, 20(1-2):3-50, 1993.

[5] P. Giorgini, J. Mylopoulos, E. Nicchiarelli, and R. Sebastiani. Reasoning with goal models. In 21st International Conference on conceptual Modeling (ER2002). Springer, 2002.

[6] P. Giorgini, J. Mylopoulos, E. Nicchiarelli, and R. Sebastiani. Formal reasoning techniques for goal models. Journal of Data Semantics, 2004.

[7] P. Giorgini, J. Mylopoulos, and R. Sebastiani. Simple and minimum-cost satisfiability for goal models.

[8] J. Gordijn and H. Akkermans. Value-based requirements engineering: exploring innovative e-commerce ideas. Requirements Engineering, 8:114-134, 2003.

[9] M. B. Holbrook. Consumer Value: A Framework for Analysis and Research. Routledge, New York, NY, 1999.

[10] C. T. Horngren and G. Foster. Cost Accounting: A Managerial Emphasis, sixth edition. Prentice-Hall, Englewood Cliffs, NJ, 1987.

[11] M. Jackson. Software Requirements and Specifications: A lexicon of practice, principles and prejudices. AddisonWesley, 1995.

[12] E. Kavakli and L. P. Goal driven requirements engineering: Analysis and critique of current methods. In T. H. John Krogstie and K. Siau, editors, Information Modeling Methods and Methodologies (Adv. Topics of Database Research), pages 102 - 124. IDEA Group, 2004.

[13] A. v. Lamsweerde, R. Darimont, and E. Letier. Managing conflicts in goal-driven requirements engineering. IEEE Transactions on Software Engineering, 24(11):908926, November 1998.

[14] J. Mylopoulos, L. Chung, and E. Yu. From object-oriented to goal-oriented requirements analysis. Communications of the ACM, 42(1):31-37, January 1999.

[15] A. Osterwalder. The Business Model Ontology - a proposition in a design science approach. $\mathrm{PhD}$ thesis, University of Lausanne, Lausanne, CH, 2004.

[16] M. E. Porter. Strategy and the Internet. Harvard Business Review, (march):63-78, 2001.

[17] M. E. Porter and V. E. Millar. How information gives you competitive advantage. Harvard Business Review, (julyaugust):149-160, 1985.

[18] D. Tapscott, D. Ticoll, and A. Lowy. Digital Capital - Harnessing the Power of Business Webs. Nicholas Brealy Publishing, London, UK, 2000.

[19] B. van der Raadt, J. Gordijn, and E. Yu. Exploring web services ideas from a business value perspective. In J. Atlee and C. Roland, editors, Proceedings of the 2005 13th IEEE International Conference on Requirements Engineering (RE05), pages 53-62, Los Alamitos, CA, 2005. IEEE CS.

[20] P. Weill and M. R. Vitale. Place to Space: Migrating to eBusiness Models. Harvard Business School Press, May 28, 2001. ISBN: 1-57851-245-X.

[21] E. Yu and J. Mylopoulos. Using goals, rules, and methods to support reasoning in business process reengineering. International Journal of Intelligent Systems in Accounting, Finance and Management, 5(1):1-13, January 1996. Special issue on Artificial Intelligence in Business Process Reengineering. 